\title{
Conteúdo polifenólico e atividade antioxidante dos frutos da palmeira Juçara (Euterpe edulis Martius)
}

\author{
LIMA, C.P. ${ }^{1 *}$; CUNICO, M.M. ${ }^{1}$; MIYAZAKI, C.M.S. ${ }^{1}$; MIGUEL, O.G. ${ }^{1}$; CÔCCO, L.C. ${ }^{3}$; YAMAMOTO, C.I. ${ }^{3}$; MIGUEL, \\ M.D. ${ }^{2}$ \\ ${ }^{1}$ Departamento de Farmácia, Laboratório de Fitoquímica; ${ }^{2}$ Laboratório de Farmacotécnica; ${ }^{3}$ Departamento de \\ Engenharia Química, Laboratório de Combustíveis Automotivos (LACAUTets), Universidade Federal do Paraná, \\ Avenida Prefeito Lothário Meissner, 632 - Jardim Botânico, CEP: 80210-170, Curitiba-Brasil ${ }^{*}$ cristinapeitz@ \\ hotmail.com
}

\begin{abstract}
RESUMO: O conteúdo polifenólico e a atividade antioxidante do extrato do fruto de Euterpe edulis Martius foram avaliados. Esta espécie é uma palmeira conhecida como Juçara, nativa da Mata Atlântica e utilizada para a extração de palmito. O processo de extração do palmito acarreta a morte da planta, uma vez que esta apresenta estipe único. A elevada demanda ocasionou a escassez deste recurso natural. Muitas espécies da Mata Atlântica podem ser utilizadas pelo manejo sustentável para a preservação e exploração econômica pelas comunidades locais. $O$ fruto da palmeira Juçara pode ser uma das alternativas de manejo sustentável dos recursos naturais da Mata Atlântica. A capacidade antioxidante do fruto pode ser utilizada como justificativa para a aplicação como alimento nutricional. O conteúdo polifenólico do fruto foi determinado pelo método de Folin-Ciocalteau e os resultados obtidos foram: 10,31 $\pm 0,25 \%, 12,42 \pm 0,89 \%, 12,75$ $\pm 0,94 \%$, para o extrato bruto, fração acetato de etila e fração remanescente, respectivamente. A atividade antioxidante foi determinada pelos métodos de redução do complexo fosfomolibdênico e DPPH. O extrato bruto e as frações acetato de etila e remanescente apresentaram atividade antioxidante, sendo que as duas últimas demonstraram maior atividade indicando que o conteúdo polifenólico pode ser responsável por esta atividade.
\end{abstract}

Palavras-chave: Euterpe edulis, Mata Atlântica, manejo sustentável, antioxidante, polifenóis

ABSTRACT: Polyphenolic content and antioxidant capacity of fruits of Juçara (Euterpe edulis Martius) palm tree. The polyphenolic content and the antioxidant activity of Euterpe edulis Martius fruit extract were assessed. This species is a Palm tree known as Juçara, native to Atlantic Forest and used for palm heart extraction. The process of palm heart extraction leads to the death of the plant since the latter has one single stem. The high demand has resulted in the depletion of this natural resource. Many species from Atlantic Forest can be used by means of sustainable management for the preservation and economic exploration by local communities. The fruit of Juçara palm can be one of the sustainable management alternatives for the Atlantic Forest natural resources. The antioxidant capacity of this fruit can justify its application as nutritional food. The fruit polyphenolic content was determined by the Folin-Ciocalteau method and the obtained results were: $10.31 \pm 0.25 \%, 12.42 \pm 0.89 \%, 12.75 \pm 0.94 \%$ for crude extract, acetyl acetate and remaining fractions, respectively. The antioxidant capacity was determined through the phosphomolybdenium complex and DPPH methods. The crude extract and the acetyl acetate and remaining fractions showed antioxidant activity, and the latter two showed higher activity, indicating that polyphenolic content may be responsible for this activity.

Key words: Euterpe edulis, Atlantic Forest, sustainable management, antioxidant, polyphenols

\section{INTRODUÇÃO}

Atualmente, o palmito oriundo da palmeira juçara, Euterpe edulis Martius é um dos produtos mais explorados na Mata Atlântica. Essa exploração tem contribuído para a degradação do meio ambiente e tornou-se fator de preocupação para a preservação da espécie, uma vez que não há rebrota após o corte

Recebido para publicação em 18/10/2010

Aceito para publicação em 28/11/2011

Rev. Bras. PI. Med., Botucatu, v.14, n.2, p.321-326, 2012. 
para a extração do palmito (Mortara \& Valeriano, 2001).

Euterpe edulis Martius, também conhecido como palmito branco, palmito verde, palmito doce e juçara, apresenta larga distribuição pelo país, do sul da Bahia até o norte do Rio Grande do Sul, sendo de ocorrência natural na Mata Tropical Atlântica do Brasil (Lima et al., 2008). Apesar de ser exigido plano de manejo sustentável para sua exploração, os casos de extração ilegal vêm aumentando (Cerisola et al., 2007).

Um dos maiores desafios do homem tem sido utilizar de forma equilibrada os recursos naturais. Tirando da natureza os meios para o sustento e desenvolvimento, o homem, com frequência, provoca intensa degradação ambiental comprometendo a vida futura. A destruição das florestas tornou-se fato tão corriqueiro que recuperar áreas degradadas é trabalho cada vez mais importante e urgente. Na MataAtlântica, reduzida a apenas $7,3 \%$ da área original, a situação é ainda mais crítica (Reis et al., 1999).

$\mathrm{Na}$ Amazônia e em outras regiões tropicais, o extrativismo tem sido algumas vezes, praticado junto com o manejo sustentado de recursos naturais; permitindo que espécies de maior utilidade econômica sejam conservadas e aproveitadas. Um caso comum de extrativismo e manejo sustentado ocorre nas matas de várzea do estuário amazônico, onde a palmeira açaí (Euterpe oleracea Martius) é uma das espécies mais abundantes e economicamente promissoras (Jardim \& Anderson, 1987).

Euterpe oleracea, o açaí, é aproveitado pelos habitantes em todos os componentes (raízes, estipes, folhas, inflorescência, palmito e principalmente os frutos). Os frutos do açaí são processados para a obtenção da polpa, que é utilizada como alimento e exportada para todo o mundo. $O$ fruto da palmeira juçara, E. edulis, é muito semelhante ao fruto do açaí. Constitui-se de drupa esférica com pericarpo pouco espesso e liso. A coloração passa do verde ao roxo negro durante a maturação. A frutificação dessa palmeira ocorre de maneira abundante entre os meses de março e junho (Cerisola et al., 2007).

Aproximadamente 10.000 toneladas de polpa de açaí são consumidas no Brasil e 1.000 toneladas exportadas para países como Japão, Estados Unidos, Holanda e Itália (Rocha et al., 2007). A polpa deste fruto tem sido objeto de estudos em função do valor nutritivo, sendo considerado um alimento nutracêutico face ao elevado teor de antocianinas, pigmentos hidrossolúveis responsáveis pela cor avermelhada do fruto (Menezes et al., 2008). O conteúdo de antocianinas é 33 vezes mais alto do que no vinho tinto, apresenta também outros benefícios como o perfil de aminoácidos e o conteúdo mineral. O açaí tem levantado a atenção principalmente nos Estados Unidos devido a presença de polifenóis e os ácidos graxos omega-6 e omega-9 (Gruenwald, 2007).

O açaí demonstra benefícios à saúde associados com a elevada capacidade antioxidante e pela composição fitoquímica (Pacheco-Palencia et al., 2008). Estudos relatam atividade antioxidante, efeito vasodilatador, inibição das enzimas COX-1 e 2 (Chin et al., 2008) e inibição da produção de NO e da expressão da enzima óxido nítrico sintetase (Matheus et al., 2006). Em estudo com a polpa de açaí foi verificada elevada ação antioxidante sobre os ânions superóxido e radical peroxila (Schauss et al., 2006). A polpa de açaí apresentou elevada atividade antioxidante em ensaio de inibição da oxidação de lipossomas (Hassimotto et al., 2005). Em estudo com voluntários humanos, o consumo de suco e polpa de açaí causou aumento de 2,3 e 3 vezes na capacidade antioxidante do plasma, respectivamente (MertensTalcott et al., 2008).

Os radicais livres causam várias doenças como arteriosclerose, câncer, Alzheimer e Parkinson, além do processo de envelhecimento. Há diversas evidencias de que o consumo de antioxidantes pode ajudar a manter a saúde e prevenir doenças (Rodrigues et al., 2006). O estresse oxidativo produz danos em lipídeos, proteínas, carboidratos e ácidos nucléicos contribuindo para problemas patológicos no organismo (Hassimotto et al., 2005). O consumo de frutas está associado com a diminuição do risco de doenças, como câncer, aterosclerose, e doenças neurodegenerativas, as quais estão associadas a elevados níveis de estresse oxidativo. Compostos antioxidantes podem minimizar estas doenças. Frutas apresentam vários compostos antioxidantes como vitamina $C, \beta$-caroteno e polifenóis (Spada et al., 2008). Os polifenóis estão correlacionados com aumento do potencial antioxidante no plasma e são reconhecidos pela proteção vascular (Mertens-Talcott et al., 2008).

A Mata Atlântica é uma das áreas do planeta que apresenta maior biodiversidade. Por motivos econômicos e históricos houve exploração demasiada dos recursos naturais, levando hoje a uma situação crítica de conservação desta biodiversidade. A redução contínua da mesma pode causar prejuízos ambientais, uma vez que as espécies presentes nos biomas estão interligadas por mecanismos naturais. O desequilíbrio das espécies pode ocasionar importantes danos nos ecossistemas como a falta de regulação do clima, desproteção dos solos contra a erosão e de bacias hidrográficas contra o assoreamento, até mesmo o aparecimento de pragas. Desta forma o manejo sustentável dos recursos naturais presentes na Mata Atlântica pode ser uma alternativa para a preservação e geração de emprego e renda para a população local.

Sendo o fruto do açaizeiro (E. oleracea) uma

Rev. Bras. PI. Med., Botucatu, v.14, n.2, p.321-326, 2012. 
fonte de renda sustentável na região amazônica, e reconhecido por suas propriedades nutricionais e antioxidantes, a utilização do fruto da palmeira juçara da mesma forma que o açaí, na forma de polpa, como alternativa comercial e para o manejo sustentável da espécie, apresenta-se como uma opção atraente. $O$ objetivo do presente trabalho foi verificar se o fruto da palmeira juçara ( $E$. edulis) apresenta, assim como o fruto do açaí, atividade antioxidante, e avaliar se esta atividade antioxidante também como no açaí está associada ao conteúdo polifenólico.

\section{MATERIAL E MÉTODO}

\section{Procedimentos experimentais gerais}

Todos os solventes utilizados foram analiticamente puros. Os padrões de ácido ascórbico, rutina e catequina foram adquiridos da Merck e oDPPH (2,2-difenil-1-picril-hidrazila) foi adquirido da Sigma. As medidas de absorção foram realizadas em espectrofotômetro UV-1601 Shimadzu.

\section{Obtenção dos extratos}

Os frutos foram coletados no município de Garuva, no estado de Santa Catarina. Os frutos foram imersos em água destilada e despolpados. A polpa foi extraída por maceração em mistura de etanol e água, por duas semanas. Após, a polpa foi filtrada e o extrato obtido foi concentrado. Na sequência, o extrato concentrado foi submetido à partição com solventes de polaridade crescente (hexano, clorofórmio e acetato de etila). A fração remanescente foi considerada o extrato aquoso obtido após a partição com todos os solventes.

\section{Determinação da atividade antioxidante}

Análise quantitativa da atividade antioxidante pelo método de redução do complexo fosfomolibdênico

A capacidade antioxidante total determinada pelo método da redução do complexo fosfomolibdênico baseia-se na determinação espectrofotométrica da redução do $\mathrm{Mo}^{+4}$ a Mo+5 , com formação subsequente de fosfato de $\mathrm{Mo}^{+5}$, que apresenta absorção máxima a $695 \mathrm{~nm}$.

Foram preparadas soluções etanólicas ou aquosas de $0,2 \mathrm{mg} \mathrm{mL}^{-1}$ dos extratos e dos padrões de ácido ascórbico e rutina. Uma alíquota de $0,3 \mathrm{~mL}$ foi colocada em tubo de ensaio, $1 \mathrm{~mL}$ de solução reagente do complexo fosfomolibdênico e $1,5 \mathrm{~mL}$ de água destilada foram adicionados no mesmo tubo. Os tubos foram incubados por $90 \mathrm{~min}$ a $95^{\circ} \mathrm{C}$ e depois resfriados até temperatura ambiente. A leitura foi realizada em espectrofotômetro em $695 \mathrm{~nm}$.

Os resultados da avaliação da atividade antioxidante foram expressos em termos percentuais na forma de atividade antioxidante relativa AAR\% (ácido ascórbico) e AAR\% (rutina). Os cálculos foram estabelecidos por meio das equações:

$\mathrm{AAR} \%$ (ac. asc.) $=($ Abs amostra - Abs branco/ Abs ác. ásc.- Abs branco) $\times 100$

AAR\% (rutina) $=($ Abs amostra - Abs branco $/$ Abs rutina - Abs branco) $\times 100$

Análise qualitativa da atividade antioxidante pelo método do DPPH (2,2-difenil-1-picril-hidrazila)

De acordo com o método descrito por Conforti et al. (2002), o extrato bruto e as frações foram submetidos a cromatografia em camada delgada (CCD). Os extratos foram analisados por CCD usando rutina e ácido ascórbico como padrões positivos de comparação. Foram utilizadas placas de cromatografia em camada delgada (cromatofolha silicagel $60 \mathrm{~F}_{254}$ Merck), nas quais foram aplicadas as amostras. As cromatoplacas com as frações hexano e clorofórmio foram eluídas na fase móvel tolueno: acetato de etila (93:7), enquanto as cromatoplacas com o extrato bruto e as frações acetato de etila e remanescente foram eluidas com a fase móvel acetato de etila: acetona: água (25:8:2). Após a secagem, as cromatoplacas foram nebulizadas com solução a $0,4 \mathrm{mmol} \mathrm{L}^{-1}$ do radical DPPH em etanol. As placas foram observadas por 30 minutos até o aparecimento de manchas amarelas sob fundo de coloração púrpura, indicativo de possível atividade antioxidante, pela redução do DPPH.

\section{Análise quantitativa da atividade antioxidante pelo método do DPPH (2,2-difenil-1-picril-hidrazila) \\ O radical DPPH é estável na coloração} púrpura, porém quando reduzido passa a ter coloração amarela. O método está baseado na capacidade do DPPH reagir com substâncias antioxidantes doadoras de hidrogênio radicalar (Sanches-Moreno, 2002).

A avaliação quantitativa da atividade antioxidante foi realizada seguindo a metodologia descrita na literatura (Mensor et al., 2001), com pequenas modificações, monitorando-se o consumo do radical livre DPPH pelas amostras, através da medida do decréscimo da absorbância de soluções de diferentes concentrações (Sousa et al., 2007). Estas medidas foram feitas em espectrofotômetro UV-Vis no comprimento de onda $518 \mathrm{~nm}$, tendo como controles positivos rutina e ácido ascórbico. No preparo da curva, foram preparadas diluições para as amostras, sendo para o ácido ascórbico de 1 a $10 \mu \mathrm{g} \mathrm{mL}^{-1}$, para a rutina de $1,25 \mathrm{a} 10 \mu \mathrm{g} \mathrm{mL}^{-1}$, para o extrato bruto 15 a $100 \mu \mathrm{g} \mathrm{mL}^{-1}$, e para a fração remanescente de 20 a $80 \mu \mathrm{g} \mathrm{mL}^{-1}$. A 2,5 mL das diluições obtidas foi adicionado $1 \mathrm{~mL}$ de DPPH 0,3 mmoles $\mathrm{L}^{-1}$. Como branco foi utilizada a mistura de $2,5 \mathrm{~mL}$ de cada diluição com $1 \mathrm{~mL}$ de etanol, ou seja para cada diluição fez-se um branco. As reações

Rev. Bras. PI. Med., Botucatu, v.14, n.2, p.321-326, 2012. 
foram realizadas em triplicata, por $30 \mathrm{~min}$ a $25^{\circ} \mathrm{C}$. Foi preparado um controle utilizando $2,5 \mathrm{~mL}$ de etanol e $1 \mathrm{~mL}$ de solução de DPPH 0,3 mmoles $\mathrm{L}^{-1}$. Para avaliar a atividade captadora de radical, foi obtida a porcentagem de inibição (IC\%), conforme a equação: $\mathrm{IC} \%=100-[($ Abs amostra - Abs branco/ Abs controle) $] \times 100$

A determinação da $I C_{50}$, ou seja, concentração da amostra ou do padrão que causa $50 \%$ de inibição da concentração inicial de DPPH foi obtida por regressão linear dos dados obtidos experimentalmente, plotados graficamente.

\section{Determinação dos polifenóis totais}

Para o doseamento dos polifenóis foi utilizado o método de Folin Ciocalteau, no qual a mistura dos ácidos fosfowolfrámico e fosfomolíbdico em meio básico se reduz ao oxidar os compostos fenólicos, originando óxidos azuis de wolframio e molibdeno (Kuskoski et al., 2006). Uma curva padrão foi preparada com a solução de catequina nas concentrações de 10 a $40 \mu \mathrm{g}$.

Em um tubo de ensaio foram adicionados $3,6 \mathrm{~mL}$ de água destilada, $200 \mu \mathrm{L}$ do reativo de Folin Ciocalteau, e $80 \mu \mathrm{L}$ de solução etanólica da amostra. A seguir os tubos foram agitados vigorosamente e então deixados em repouso por 3 minutos. Foram adicionados $0,4 \mathrm{~mL}$ de solução de carbonato de sódio a $35 \%(\mathrm{~m} / \mathrm{v})$ em cada tubo. Os tubos foram novamente agitados e deixados em repouso durante 60 minutos. A leitura foi realizada em espectrofotômetro a 725 $\mathrm{nm}$. Com os resultados das absorbâncias, os dados foram interpolados e foi determinada a equação da reta. Os resultados foram expressos em porcentagem de polifenóis presentes.

\section{Análise Estatística}

Os resultados apresentados correspondem à média $\pm S D$ de três repetições, e foram comparados por análise de variância (ANOVA) seguido do teste de Tukey para identificar as diferenças significativas entre as médias, utilizando o programa Sisvar, onde as médias em nível de $5 \%(p<0,05)$ foram consideradas significantes.

\section{RESULTADO E DISCUSSÃO}

Na Tabela 1 são apresentados os resultados obtidos para a avaliação da atividade antioxidante relativa do extrato e das frações em relação ao ácido ascórbico e à rutina pela formação do complexo fosfomolibdênico. Observa-se que as frações acetato de etila e fração remanescente apresentam atividade antioxidante superior ao do extrato bruto. Já as frações hexano e clorofórmio apresentaram atividade antioxidante inferior àquela apresentada pelo extrato bruto. Estes resultados indicam que compostos presentes nas frações acetato de etila e remanescente são responsáveis pela atividade antioxidante verificada no extrato bruto. A fração remanescente apresentou atividade antioxidante em relação à rutina superior a $50 \%$. O padrão rutina demonstrou atividade antioxidante em relação ao ácido ascórbico de 26,66 $\pm 0,45 \%$. A avaliação da capacidade antioxidante pelo método da formação do complexo fosfomolibdênico é mais adequada para substâncias como a vitamina C (ácido ascórbico) e E. Para compostos fenólicos como a rutina e os presentes na fração acetato de etila e remanescente, outros métodos devem ser utilizados para a avaliação da atividade antioxidante.

Os resultados da avaliação preliminar do extrato e das frações por CCD em sílica gel, revelada com solução etanólica de DPPH $0,4 \mathrm{mmol} \mathrm{L}^{-1}$, sugeriu a presença de substâncias com atividade antioxidante no extrato bruto e nas frações acetato de etila e remanescente, pela presença de manchas amarelas na cromatoplaca, resultantes da ação antioxidante sobre o DPPH. Na Tabela 2, observa-se a quantidade de extrato, frações e dos padrões ácido ascórbico e rutina necessários para reduzir a concentração de DPPH em $50 \%\left(\mathrm{IC}_{50}\right)$. As frações hexano e clorofórmio apresentaram IC ${ }_{50}$ superior a $1000 \mu \mathrm{g} \mathrm{mL}^{-1}$, portanto não demonstraram atividade antioxidante neste ensaio. O extrato bruto apresentou $\mathrm{IC}_{50}$ de 67,95 \pm $0,25 \mu \mathrm{g} \mathrm{mL} \mathrm{mL}^{-1}$ e as frações acetato de etila e remanescente mostraram, assim como no ensaio da formação do complexo fosfomolibdênico atividade antioxidante superior ao do extrato bruto, resultando nos valores de $\mathrm{IC}_{50} 50,7 \pm 0,98$ e 43,64 $\mu \mathrm{g} \mathrm{mL}^{-1}$, respectivamente.

TABELA 1. Atividade antioxidante relativa (\%) do extrato e das frações em relação aos padrões vitamina C e rutina, pela formação do complexo fosfomolibdênico.

\begin{tabular}{lcc}
\hline Amostra & AAR\% ácido ascórbicox $\pm \boldsymbol{d p}$ & AAR\% rutinax $\pm \boldsymbol{d p}$ \\
\hline Extrato bruto & $13,47 \pm 0,08 \mathrm{c}$ & $41,73 \pm 0,17 \mathrm{c}$ \\
Fração Hexano & $10,48 \pm 0,22 \mathrm{~b}$ & $40,40 \pm 1,09 \mathrm{~b}$ \\
Fração Clorofórmio & $8,87 \pm 0,61 \mathrm{a}$ & $33,32 \pm 0,62 \mathrm{a}$ \\
Fração Acetato de Etila & $14,09 \pm 0,39 \mathrm{~d}$ & $48,87 \pm 1,67 \mathrm{~d}$ \\
Fração Remanescente & $21,62 \pm 0,15 \mathrm{e}$ & $66,94 \pm 0,55 \mathrm{e}$ \\
\hline
\end{tabular}

Valores seguidos de letras diferentes na mesma coluna diferem estatisticamente entre si. 
Em estudo realizado por Pacheco-Palencia et al. (2009), com diferentes tipos de óleo de açaí, foi verificado que o óleo rico em compostos fenólicos, como os ácidos protocatequico, $p$-hidroxibenzóico, vanilíco, siríngico, ferúlico, catequina, e dímeros e trímeros de procianidinas, obtido de um produto da manufatura de suco de açaí, apresentou, em comparação com outros dois óleos (um, de composição intermediária, e outro, de composição baixa em compostos fenólicos), maior atividade antioxidante que os outros dois. Da mesma forma, os resultados obtidos no presente ensaio também confirmam que as frações com maior conteúdo de compostos fenólicos apresentam maior atividade antioxidante. Em outro ensaio Seeram et al. (2008), analisando diferentes marcas comerciais de bebidas de frutas com elevados teores de compostos polifenólicos, verificaram que o aumento do potencial antioxidante se correlaciona com o aumento da quantidade de polifenóis.

Os compostos fenólicos são os principais responsáveis pela atividade antioxidante em frutos (Heim et al., 2002). As polpas de frutas como amora, açaí, uva, goiaba, analisados quanto à atividade antioxidante pelo método do DPPH, mostraram que os compostos fenólicos contribuem para a atividade antioxidante, apresentando correlação direta. Silva et al. (2007), estudando 15 espécies de plantas da Amazônia, obtiveram correlação positiva entre polifenóis e atividade antioxidante.

A quantidade de moléculas de DPPH que é reduzida está correlacionada com a presença de grupos hidroxilas (Mensor et al., 2001). Em estudo com taninos condensados das folhas de Maytenus ilicifolia, realizado por Pessuto et al. (2009), foi demonstrado que quanto maior o número de hidroxilas fenólicas, maior a capacidade de sequestro de radicais livres.

A correlação entre fenóis totais e a capacidade antioxidante pode depender do método

TABELA 2. Atividade antioxidante do extrato, das frações e dos padrões ácido ascórbico e rutina pela redução do radical $\mathrm{DPPH}\left(\mathrm{IC}_{50}\right)$ e teor de polifenóis totais (PT\%).

\begin{tabular}{lcc}
\hline Amostra & $\begin{array}{c}\mathbf{I C}_{\mathbf{5 0}}\left(\mathbf{m g ~ m L}^{-1}\right) \\
\mathbf{x} \mathbf{d} \mathbf{d p}\end{array}$ & $\begin{array}{c}\mathbf{P T} \% \\
\mathbf{x} \pm \mathbf{d p}\end{array}$ \\
\hline Extrato bruto & $67,95 \pm 0,25 \mathrm{e}$ & $10,31 \pm 0,25 \mathrm{a}$ \\
Fração Hexano & $>1000$ & $\mathrm{nd}$ \\
Fração Clorofórmio & $>1000$ & $\mathrm{nd}$ \\
Fração Acetato de Etila & $50,7 \pm 0,98 \mathrm{~d}$ & $12,42 \pm 0,89 \mathrm{~b}$ \\
Fração Remanescente & $43,64 \pm 0,21 \mathrm{c}$ & $12,75 \pm 0,94 \mathrm{c}$ \\
Acido ascórbico & $6,13 \pm 0,05 \mathrm{a}$ & - \\
Rutina & $6,71 \pm 0,03 \mathrm{~b}$ & - \\
\hline
\end{tabular}

(1) Valores seguidos de letras diferentes na mesma coluna diferem estatisticamente entre si. nd = não detectado escolhido, e também das características hidrofóbicas e ou hidrofílicas do sistema teste e dos antioxidantes testados (Roesler et al., 2007). A capacidade antioxidante dos compostos fenólicos depende de diversos fatores, como sistema oxidante, grau de glicosilação e coeficiente de partição (Hassimatto et al., 2005).

O estresse oxidativo é causado pela insuficiência da capacidade dos sistemas biológicos em neutralizar o excesso de produção de radicais livres. Estes radicais podem contribuir para o aparecimento de doenças neurodegenerativas e cardiovasculares, obesidade e resistência a insulina, além de envelhecimento (Jensen et al., 2008). Estudos indicam que pessoas que ingerem dieta rica em frutas e vegetais, reduzem a incidência de doenças associadas ao envelhecimento (Stevenson et al., 2007). A proteção das frutas e vegetais contra estas doenças é atribuída aos vários antioxidantes nelas contidas (Wang et al., 1996). O chamado "Paradoxo Francês" também tem sido atribuído em parte ao consumo rotineiro de vinho tinto o qual possui abundante quantidade de polifenóis (Hassimotto et al., 2005). Estes resultados indicam o potencial de uso da polpa dos frutos da palmeira Juçara, com o propósito de alimento com propriedades antioxidantes.

A correlação entre o consumo de compostos polifenólicos e a melhora na saúde tem sido relatada em diversos estudos (Nichenametla et al., 2006). Dieta rica em frutas como o açaí que contém elevados teores de polifenóis proporciona prevenção contra doenças, devido às propriedades antioxidantes. Por estas propriedades, nos últimos anos, o açaí tem sido utilizado no mercado nacional e internacional como alimento funcional. Da mesma forma que o açaí, o fruto da palmeira Juçara pode ser utilizado como alimento funcional, devido às propriedades antioxidantes. Possibilitando assim o uso de maneira sustentável, uma vez que esta espécie está ameaçada de extinção devido à exploração predatória de palmito.

A Mata Atlântica é um dos 25 hotspots mundiais de biodiversidade. Embora tenha sido em grande parte destruída, ainda abriga mais de 8.000 espécies endêmicas de plantas vasculares, anfíbios, répteis, aves e mamíferos (Tabarelli et al., 2005). A conservação deste ecossistema é um desafio, pois está sob forte pressão antrópica. Por isso faz-se necessário medidas de proteção e de manejo sustentável deste bioma. Assim, o uso da polpa do fruto da palmeira Juçara como alimento torna-se uma alternativa de exploração sustentável.

\section{AGRADECIMENTO}

Os autores agradecem a CAPES pela bolsa concedida a Cristina Peitz de Lima e a Andrey Pabst, da empresa Alicon Alimentos, pelo fornecimento do material vegetal. 


\section{REFERÊNCIA}

CERISOLA, C.M.;ANTUNES, A.Z.; PORT-CARVALHO, M. Consumo de frutos de Euterpe edulis Martius (Arecaceae) por vertebrados no Parque Estadual Alberto Löfgren, São Paulo, Sudeste do Brasil. Revista do Instituto Florestal e IF Série Registros, n.31, p.167-71, 2007.

$\mathrm{CHIN}, \mathrm{Y}$. et al. Lignans and other constituents of the fruits of Euterpe oleracea (açai) with antioxidant and cytoprotective activities. Journal of Agricultural and Food Chemistry, v.56, p.7759-64, 2008.

CONFORTI, F. et al. Antioxidant activity of methanolic extract of Hypericum triquetrifoium turra aerial part. Fitoterapia, v.73, n.6, p.479-83, 2002.

GRUENWALD, J. Novel botanical ingredients for beverages. Clinics in Dermatology, v.27, p.210-6, 2009. HASSIMOTTO, N.M.A.; GENOVESE, M.I.; LAJOLO, F.M. Antioxidant activity of dietary fruits, vegetables, and commercial frozen fruit pulps. Journal of Agricultural and Food Chemistry, v.53, n.8, p.2928-35, 2005.

HEIM, K.E.; TAGLIAFERRO, A.R.; BOBILYA, D.J. Flavonoid antioxidants: chemistry, metabolism and structure activity relationships. The Journal of Nutritional Biochemistry, v.13, p.572-84, 2002.

JARDIM, M.A.G.; ANDERSON, A.B. Manejo de populações nativas de açaizeiro no estuário amazônico resultados preliminares. Boletim de Pesquisa Florestal, n.15, p.1$18,1987$.

JENSEN, G.S. et al. In vitro and in vivo antioxidant and anti-inflammatory capacities of an antioxidant-rich fruit and berry juice blend. Results of a pilot and randomized, double-blinded, placebo-controlled, crossover study. Journal of Agricultural and Food Chemistry, v.56, n.18, p.8326-33, 2008.

KUSKOSKI, E.M. et al. Frutos tropicais silvestres e polpas de frutas congeladas: atividade antioxidante, polifenóis e antocianinas. Ciência Rural, v.36, n.4, p.1283-7, 2006. LIMA, L.S.H.; FRANCO, E.T.H.; SCHUMACHER, M.V. Crescimento de mudas de Euterpe edulis Martius em resposta a diferentes doses de fósforo. Ciência Florestal, v.18, n.4, p.461-70, 2008.

MATHEUS, M.E. et al. Inhibitory effects of Euterpe edulis Mart. on nitric oxide production and iNOS expression. Journal of Ethno pharmacology, v.107, p.291-6, 2006. MENEZES, E.M.S.; TORRES, A.T.; SRUR, A.U.S. Valor nutricional da polpa de açaí (Euterpe oleracea Mart) liofilizada. Acta Amazônica, v.38, n.2, p.311-6, 2008.

MENSOR, L.L. et al. Screening of brazilian plant extracts for antioxidant activity by the use of DPPH free radical method. Phytotherapy Research, v.15, p.127-30, 2001. MERTENS-TALCOTT, S.U. et al. Pharmacokinetics of anthocyanins and antioxidant effects after the consumption of anthocyanin-rich açai juice and pulp (Euterpe oleracea Mart.) in human healthy volunteers. Journal of Agricultural and Food Chemistry, v.56, p.7796-802, 2008.

MORTARA, M.O.; VALERIANO, D.M. Modelagem da distribuição potencial do palmiteiro (Euterpe edulis Martius) a partir de variáveis topográficas. In: SIMPÓSIO BRASILEIRO DE SENSORIAMENTO REMOTO, 10., 2001, Foz do Iguaçu. Resumos... Foz do Iguaçu: INEPE,
2001. p.459-71.

NICHENAMETLA, S.N. et al. A review of the effects and mechanisms of polyphenolics in cancer. Critical Reviews in Food Science and Nutrition, v.46, n.2, p.161-83, 2006. PACHECO-PALENCIA, L.A.; MERTENS-TALCOTT, S.; TALCOTT, S.T. Chemical composition, antioxidant properties, and thermal stability of a phytochemical enriched oil from açai (Euterpe oleracea Mart.). Journal of Agricultural and Food Chemistry, v.56, n.12, p.46316, 2008.

PESSUTO, M.B. et al. Atividade antioxidante de extratos e taninos condensados das folhas de Maytenus ilicifolia Mart. ex Reiss. Química Nova, v.32, n.2, p.412-6, 2009. REIS, A.; ZAMBONIN, R.M.; NAKAZONO, E.M. Recuperação de áreas florestais degradadas utilizando a sucessão e as interações planta-animal. São Paulo: Conselho Nacional da Reserva da Biosfera da Mata Atlântica, 1999. 42p.

ROCHA, A.P.M. et al. Endothelium-dependent vasodilator effect of Euterpe oleracea Mart. (Açaí) extracts in mesenteric vascular bed of the rat. Vascular Pharmacology, v.46, n.2, p.97-107, 2007.

RODRIGUES, R.B. et al. Total oxidant scavenging capacity of Euterpe oleracea Mart. (açai) seeds and Identification of their polyphenolic compounds. Journal of Agricultural and Food Chemistry, v.54, n.12, p.41627, 2006.

ROESLER, R. et al. Atividade antioxidante de frutas do cerrado. Ciência e Tecnologia de Alimentos, v.27, n.1, p.53-60, 2007.

SÁNCHES-MORENO, C. Review: methods used evaluate the free radical scavenging activity in foods and biological systems. Food Science and Technology International, v.8, p.121-37, 2002.

SCHAUSS, A.G. et al. Phytochemical and nutrient composition of the freeze-dried amazonian palm berry, Euterpe oleraceae Mart. (Acai). Journal of Agricultural and Food Chemistry, v.54, n.22, p.8598-603, 2006.

SILVA, E.M. et al. Antioxidant activities and polyphenolic contents of fifteen selected plant species from the Amazonian region. Food Chemistry, v.101, p.1012-8, 2007. SPADA, P.D.S. et al. Antioxidant, mutagenic, and antimutagenic activity of frozen fruits. Journal of Medicinal Food, v.11, n.1, p.144-51, 2008.

SEERAM, N.P. et al. Comparison of antioxidant potency of commonly consumed polyphenol-Rich Beverages in the United States. Journal of Agricultural and Food Chemistry, v.56, n.4, p.1415-22, 2008.

SOUSA, C.M.M. et al. Fenóis totais e atividade antioxidante de cinco plantas medicinais. Química Nova, v.30, n.2, p.351-5, 2007.

STEVENSON, D.E.; HURST, R.D. Polyphenolic phytochemicals - just antioxidants or much more? Cellular and Molecular Life Sciences, v.64, p.290016, 2007.

WANG, H.; CAO, G.; PRIOR, R.L. Total antioxidant capacity of fruits. Journal of Agricultural and Food Chemistry, v.44, n.3, p.701-5, 1996.

TABARELLI, M. et al. Desafios e oportunidades para a conservação da biodiversidade na Mata Atlântica brasileira. Megadiversidade, v.1, n.1, p.132-8, 2005. 